\title{
ARTIFICIAL INTELLIGENCE TECHNIQUES FOR PREDICTING TIDAL EFFECTS BASED ON GEOGRAPHIC LOCATIONS IN GHANA
}

\author{
Yakubu ISSAKA (®*, Bernard KUMI-BOATENG ${ }^{\circledR}$ \\ Geomatic Engineering Department, University of Mines and Technology, Tarkwa, Ghana
}

Received 15 January 2019; accepted 27 February 2020

\begin{abstract}
Tidal forces as a result of attraction of external bodies (Sun, Moon and Stars) through gravity and are a source of noise in many geoscientific field observations. The solid earth tides cause deformation. This deformation results in displacement in geographic positions on the surface of the earth. The displacement due to tidal effects can result in deformation of engineering structures, loss of lives, and economic cost. Tidal forces also help in detecting other environmental and tectonic signals. This study quantifies the effects of solid earth tides on stationary survey controls in five regions of Ghana. The study is in two stages: firstly, the solid earth tides were estimated for each control by a geometric approach (combining Navier's equation of motion and Love theories). Secondly, estimation using two artificial intelligence methods (Multivariate Adaptive Regression Splines (MARS) and Backpropagation Artificial Neural Network (ANN)). Based on statistical indices of Mean Square Error (MSE) and Correlation Coefficient (R), BPANN, and MARS models can be used as a realistic alternative technique in quantifying solid earth tides for the study area. The MSE and R (MSE; BPANN $=1.3249 \times 10^{-04}$ and MSE; MARS $=2.2052 \times 10^{-06} ; \mathrm{R} ; \mathrm{BPANN}=-0.6067$ and R; MARS 0.6570$)$ values indicate that MARS outperforms BPANN in quantifying solid earth tides in the study area. BPANN and MARS can be used as an efficient tool for quantifying tidal values based on geographic positions for geodetic deformation studies within the study area.
\end{abstract}

Keywords: tidal effects, backpropagation artificial neural network, multivariate adaptive regression splines, geodetic deformation.

\section{Introduction}

Earth tides movement within the solid earth is caused by the tidal forces from external bodies. In geodetic and survey measurements, there have been an increasing demand for precision and accuracy, hence the need to determine tidal effects on the surface of the earth and apply correction (Agnew, 2007). The frequent occurrence of disasters worldwide has incited the need for rigorous experimentation with advanced techniques that would help mitigate risk (Straser, 2010), as these disasters results in countless loss of lives and properties, thus, increasing the economic expenditure of nations. The mechanisms that triggers the earth deformation is being incited by lunar/solar gravitational interaction. In literature, several studies have been conducted to determine the relationship between lunar cycles and terrestrial cycles utilizing various mathematical models, but however are not yet conclusive (Kolvankar et al., 2010). These studies applied both numerical analysis and the usage of high sensor instruments for studying the tidal effects on the earth crust for geodetic deformation modelling. Notable among these are the use of Global
Positioning System (GPS) (Yakubu, 2008; Rafiq \& Santos, 2004), Advanced Laser Interferometer Gravitational Wave Observatory (aLIGO) (Kurinsky, 2013), Seismicity (Straser, 2010), Superconducting Gravimeters (Heping et al., 2005). Notable among the mathematical models include Numerical Analysis (Munk \& Cartwight, 1966; Siek \& Solomatine, 2010), Harmonics Analysis (Vaziri, 1997), and Interpolation techniques (Kalman, 1960). These methods are widely used in geodetic studies for tides predictions and modelling.

In this study, quantification of solid earth tides of survey control stations in five regions within Ghana was carried-out to determine how significant the values are and its implementation in geodetic deformation. $\mathrm{Nu}$ merical Analysis (Navier's equations of motion and Love theories) proposed by Torge (1991) and Kurinsky (2013) were adopted to quantify the tidal effects numerically. The study also assesses the performance of two artificial intelligence models (MARS and BPANN) as a realistic alternative technique in estimating the solid earth tides values. MARS and BPANN models which are both machine

${ }^{*}$ Corresponding author. E-mail: yissaka@umat.edu.gh 
learning techniques have the capabilities of forming linear relationships between nonlinear variables. These models were adopted due to their efficiencies and performance as indicated in existing literature. Notable literature includes tidal studies (Beltrami, 2008; Mendieta, 2001), slope stability analysis (Samui, 2013), modelling of the ionosphere (Durmaz \& Karslioglu, 2011) and geodetic datum transformation (Ziggah et al., 2016a, 2016b). These models are highly regarded for their local characters modelling capabilities (Kutoglu, 2006) and a preferred predictive tool for finding solutions to some of the problems in surveying and geodesy. In this paper, the performance indicators such as the Mean Square Error (MSE) and Correlation coefficient $(\mathrm{R})$ were used to validate the models. Modelling and predicting tides and their effect is a challenge due to the high and extremely non-linear relationship between the features that cause the deformation, hence, the need by the Authors to apply the mathematical relationship. Also, the applicability and performance valuation of these methods for quantifying tidal effect in Ghana have not been evaluated.

\section{Study area and data used}

The study area (Figure 1) formally known as Gold Coast is in West Africa and share boundaries with three neighboring countries, Cote d'Ivoire to the West, Burkina Faso to the North and Togo to the East. Ghana is administratively divided into 10 regions and 170 districts (Anane, 2015). Ghana lies within latitude $4^{\circ} 44^{\prime} \mathrm{N}$ to $11^{\circ} 11^{\prime} \mathrm{N}$, and longitude $3^{\circ} 11^{\prime} \mathrm{W}$ to $01^{\circ} 11^{\prime} \mathrm{E}$. The Gulf of Guinea of the Atlantic Ocean lies on the southern part of the country, forming a coastline of about $550 \mathrm{~km}$ long. Ghana covers a total land area of about $239000 \mathrm{~km}^{2}$. The Greenwich meridian passes through Tema near Accra making Ghana geographically the closest to the centre of the World (Mohammed, 2015). The notional point of intersection between the equator and longitude $00^{\circ}$ located in the Atlantic Ocean is about $654 \mathrm{~km}$ from Accra (Mohammed, 2015).

Data used in this paper is a secondary data obtained from the Survey and Mapping Division of the Lands Commission, Ghana. The data consist of latitude and longitude of 94 surveyed stations and covers six regions (Southern part of Ghana). Sample of the data used is shown in Table 1 . These data were used in quantifying solid earth tides for the study area.

Table 1. Sample of data used (units in degree decimals)

\begin{tabular}{|c|c|}
\hline Latitude & Longitude \\
\hline 5.66600000000 & 0.42333333330 \\
\hline 5.69666666670 & 0.31500000000 \\
\hline 5.70383333330 & 0.07933333330 \\
\hline 5.68833333330 & 0.05000000000 \\
\hline 5.82783333330 & 0.06566666670 \\
\hline 5.82000000000 & 0.18833333330 \\
\hline 5.93683333330 & 0.05216666670 \\
\hline 5.95116666670 & 0.19383333330 \\
\hline 5.92283333330 & 0.20916666670 \\
\hline 5.78666666670 & 0.43666666670 \\
\hline
\end{tabular}

\section{Methods used}

\subsection{Tidal potential}

A functional form for the tidal potential was attained by projecting the geometric potential. This yields the following potential as denoted by Equation (1) (Kurinsky, 2013;

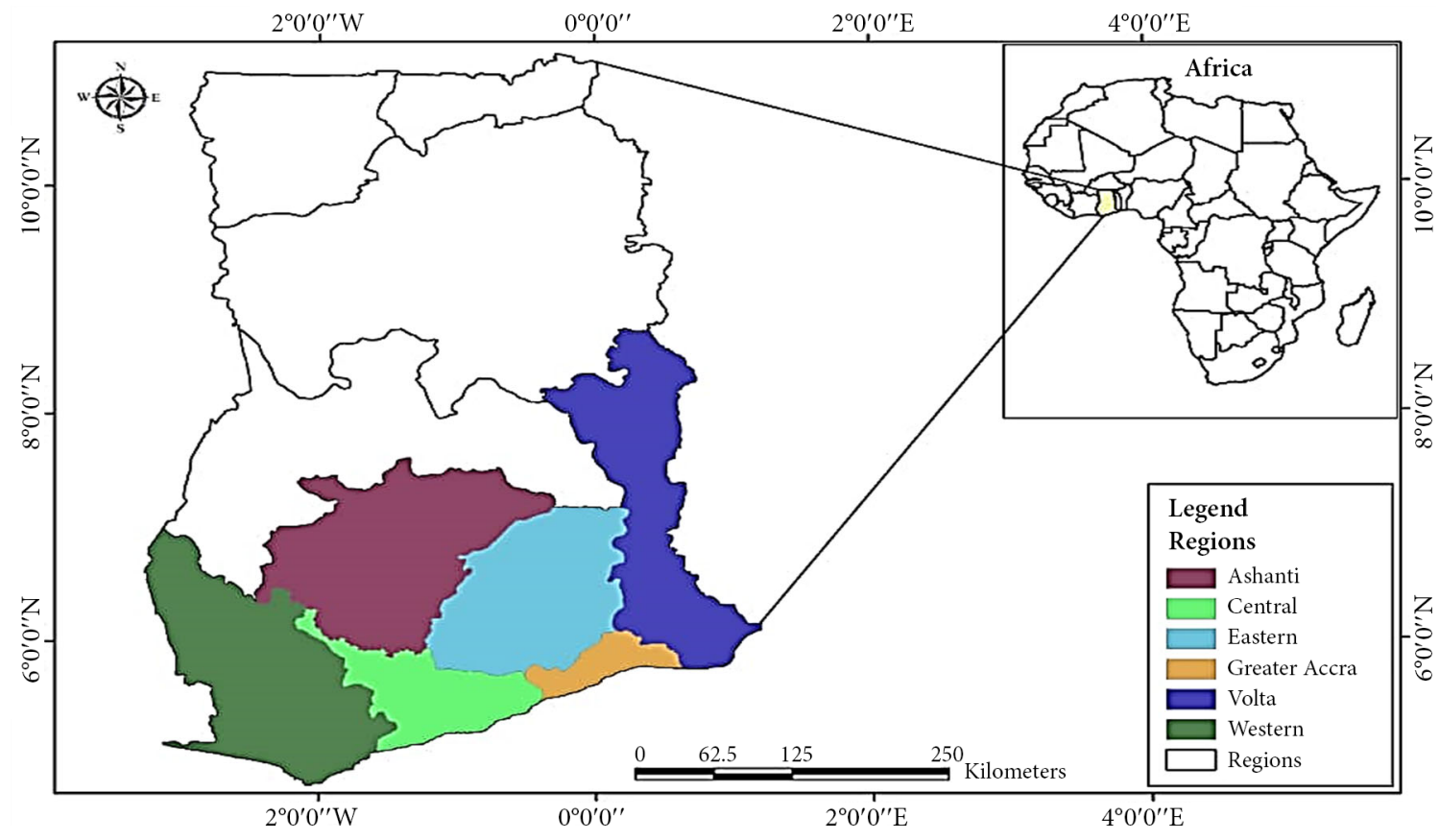

Figure 1. The Study Area Showing the Six Selected Regions of Ghana 
Torge, 1991) denoted as

$$
T(Q)=D\left(\frac{r}{a}\right)^{2}\left(\frac{p}{d}\right)^{3}\left[\begin{array}{l}
\cos ^{2} \varphi \cos ^{2} \delta \cos 2 H+ \\
\sin 2 \varphi \sin 2 \delta \cos H+ \\
3\left(\sin ^{2} \phi-\frac{1}{3}\right)\left(\sin ^{2} \delta-\frac{1}{3}\right.
\end{array}\right],
$$

where $p$ is the average object distance, $\Phi$ and $\lambda$ are the latitude angle and longitude correspondingly, $\phi=90-\Phi$ is the colatitude of the position, and $\delta$ is the declination of the external body.

The two other terms initiate here are Doodson's constant, denoted by Equation (2) (Kuirnsky, 2013) as

$$
D=\frac{3}{4} G M_{o} \frac{a^{2}}{p^{3}} \text {. }
$$

The local hour angle $\mathrm{H}$ is given by Equation (3) (Kurinsky, 2013) as

$$
H(T)=w T-\alpha-\lambda,
$$

where $w$ is the sidereal velocity of the earth, $T$ is the sidereal time and $\alpha$ is the right ascension of the body (Kurinsky, 2013).

\subsection{Love parameterization}

To employ the functional potential to model solid earth tides, a model which links the shape of the tidal potential to the earth deformation according to Equation (4) is given as (Kurinsky, 2013);

$$
X_{r}=h \frac{T(Q)}{g}, X_{\varphi}=\frac{l}{g} \frac{T(Q)}{\partial \varphi}, X_{\lambda}=\frac{l}{g \sin (\varphi)} \frac{\partial T(Q)}{\partial \lambda},
$$

where the variables are as well-defined in the potential, $g$ is the gravitational acceleration at $r \approx a$, and $l$ and $h$ are in the range $(0,1)$ with typical values $h \approx 0.06$ and $h \approx 0.08$ (Kurinsky, 2013).

\subsection{Displacement}

The tidal deformation of the earth is solved numerically. These displacements can be added to the position of the points in geocentric coordinates to obtain the new location of the points. The equations adopted include Equations (5) to (7) denoted as

$$
\begin{gathered}
X_{r}=\frac{3 h G M_{o} r^{2}}{4 g d^{3}}\left(\begin{array}{l}
\cos ^{2}(\varphi) \cos ^{2}(\delta) \cos (2 H) \\
+\sin (2 \varphi) \sin (2 \delta
\end{array}\right) \cos (H)+ \\
\left.3\left(\sin ^{2}(\varphi)-\frac{1}{3}\right)\left(\sin ^{2}(\delta)-\frac{1}{3}\right)\right) ; \\
X_{\varphi}=\frac{3 l G M_{o} r}{4 g d^{3}}\left(\begin{array}{l}
-\sin (2 \varphi) \cos ^{2}(\delta) \cos (2 H) \\
+2 \cos (2 \varphi) \sin (2 \delta) \cos (H) \\
+3 \sin (2 \varphi)\left(\sin ^{2}(\delta)-\frac{1}{3}\right)
\end{array}\right) ;
\end{gathered}
$$

$$
X_{\lambda}=\frac{3 l G M_{o} r}{4 g d^{3} \cos (\varphi)}\left(\begin{array}{l}
2 \cos ^{2}(\varphi) \cos ^{2}(\delta) \sin (2 H) \\
+\sin (2 \varphi) \sin (2 \delta) \sin (H)
\end{array}\right) .
$$

In the equations, $M_{0}$ is object mass, and all other terms are defined in the sections.

\subsection{Gravity tide}

Gravity tides show that the moon and sun exist but does not provide much information about the physical earth (Agnew, 2007). This was computed using Equation (8) denoted as

$$
\delta_{g}=\frac{g}{a} \sum_{n=i}^{M} \delta_{n} N_{i j}
$$

where $\delta_{g}$ is the gravity tide effect, $g$ is the gravitational acceleration due to gravity, a is the radius of the earth, $\delta_{n}$ is the gravimetric factor, $N_{i j}$ is calculated by the EGM2008 (Pavlis et al., 2008). Computed values from the EGM2008 model was based on the spherical harmonic equation ( $\mathrm{Lu}$ et al., 2014) as in Equation (9):

$$
V_{(p, \varphi, \lambda)}=\sum_{n=0}^{\infty} \frac{1}{p^{n+1}} \sum_{k=0}^{n}\left(\begin{array}{l}
a_{n k} \cos k \lambda+ \\
b_{n k} \sin k \lambda
\end{array}\right) p_{n k}(\cos \varphi),
$$

where $(p, \varphi, \lambda)=$ are the spherical coordinates $a_{n k}$ and $b_{n k}$ are the coefficients of the Earth's gravity field $p_{n k}(\cos \varphi)=$ represents the associated Legendre polynomials, $n$ is the degree, and $k$ is the order.

\subsection{Resultant displacement}

The resultant displacement was calculated according to Equation (10) given as

$$
d s=\sqrt{X_{r}^{2}+X_{\lambda}^{2}+X_{\phi}^{2}+\delta_{n}^{2}} .
$$

\subsection{Multivariate Adaptive Regression Splines (MARS)}

The MARS model is nonparametric (Friedman, 1991) and it works by dividing the variables into regions, producing each region a linear regression equation (Zabihi et al., 2016). Knots are the breaks values between the regions. The term basis functions (BFs) indicates the distinct interval of the predictors (Samui \& Kim, 2012). The general equation for the MARS model is given by Equation (11) (Samui, 2013):

$$
y=f(x)=a_{0}+\sum_{n=1}^{N} \alpha_{n} \beta_{n}(x),
$$

where $y$ is the dependent variable predicted by the function $f(x), a_{0}$ is a constant, and $N$ is the number of terms, each of them formed by a coefficient $\alpha_{n}$ and $\beta_{n}(x)$ is an individual basis function or a product of two or more BFs. Two steps were used to develop the MARS model. In the first case, the forward algorithm, basis functions as define Equation (11). To achieve a better estimate 
of the dependent value, many basis functions are added in Equation (1) (Zabihi et al., 2016; Samui \& Kim, 2012). Due to the large number of basis used, the MARS model may experience overfitting (Friedman, 1991). To resolve the overfitting problem, a second case which is the backward algorithm is used. Results are achieved by eliminating redundant basis functions from Equation (11). Generalized Cross-Validation (GCV) is used in the MARS model to remove redundant basis functions (Samui \& Kothari, 2012). Equation (12) GCV expression (Craven \& Wahba, 1979):

$$
G C V=\frac{\frac{1}{N} \sum_{i=1}^{N}\left[y_{i}-\dot{f}\left(x_{i}\right)\right]^{2}}{\left[1-\frac{C(H)}{N}\right]^{2}},
$$

where $N$ is the number of data and $C(H)$ is a complexity penalty that increases with the number of basis function (BFs) in the model and which is defined as denoted by Equation (13):

$$
C(H)=(h+1)+d H,
$$

where $d$ is a penalty for each BFs included into the model and $H$ is the number of basis functions in Equation (2) (Friedman, 1991; Samui \& Kothari, 2012).

\subsection{Backpropagation Artificial Neural Network (BAPNN)}

The BPANN is made up of an input layer, hidden layer(s) and an output layer. The output layer has processing neurons. Through a set of connection weight, the feeding layers has a feed forward training (Yegnanarayana, 2005). Inputting data is done at the input layer and the final results of the processing data is done at the output layer. The hidden layer is where the neurons receive the input data for processing. The number of hidden neurons is normally obtained through the sequential trial-and-error approach (Ziggah et al., 2016b). Normalization of the dataset is required in BPANN model formulation. In this paper, Equation (14) is used for the normalization of the input and output variables (Mueller \& Hemond, 2013):

$$
y_{i}=y_{\min }+\frac{\left(y_{\max }-y_{\min }\right) \times\left(x_{i}-x_{\min }\right)}{\left(x_{\max }-x_{\min }\right)},
$$

where $y_{i}$ represents the normalized data, $x_{i}$ is the measured coordinate value, while $x_{\min }$ and $x_{\max }$ represent the minimum and maximum values of the measured coordinates with $y_{\max }$ and $y_{\min }$ values set at 1 and -1 , respectively.

\subsection{Model performance assessment}

To assess the accuracy of the models, performance indicators such as the Mean Square Error (MSE) and Correlation coefficient $(\mathrm{R})$ were used as shown Equations (15) and (16) as

$$
M S E=\frac{1}{n} \sum_{i=1}^{n}\left(\alpha_{i}-\beta_{i}\right) .
$$

Here, $n$ is the total number of test examples presented to the learning algorithms, $\alpha_{i}$ and $\beta_{i}$ are the measured and predicted geoid heights from the various procedures.

$$
R=\frac{\sum_{i=1}^{N}\left(Z_{j a i}-\bar{Z}_{j a}\right)\left(Z_{j p i}-\bar{Z}_{j p}\right)}{\sqrt{\sum_{i=1}^{N}\left(Z_{j a i}-\bar{Z}_{j a}\right)} \sqrt{\left(\sum_{i=1}^{N} Z_{j p i}-\bar{Z}_{j p}\right)}},
$$

where $Z_{j a i}$ and $Z_{j p i}$ are the actual and predicted $Z_{j}$ values, respectively. $\bar{Z}_{j a}$ and $\bar{Z}_{j p}$ are the mean of actual and predicted $Z_{j}$ values corresponding to $N$ patterns. For an adequate model, the value of $R$ should be close to one (Samui, 2013).

\section{Results and discussion}

The results for the computed displacement (Table 4) using Equation (10) for the various geographic coordinates of the covered regions is represented by Figure 2. It is observed that, the external bodies combined with gravity releases forces which causes movement within the earth crust. Based on geographic location, periodic monitoring of natural and engineering structures requires the computation of tidal effects.

In the models' formulation, the independent variables were the geodetic coordinates (latitude and Longitude) denoted as $\left(\phi_{i, j}, \lambda_{i, j}\right)$ and the dependent variable was the resultant displacement denoted as $\left(d s_{i, j}\right)$ respectively. In the MARS and BPANN models' formulation, $70 \%$ of the dataset was used for training the model, and the 30\% was used as test data. The optimal solution for the BPANN model after the trial and error training was [2 17 1]. Thus, 2 input variables, 17 hidden neurons and 1 output variable. In MARS model formulation, 15 basis functions were used in the forward training. 5 basis functions were used in the final model formulation for estimating the solid earth tides. This implies that 10 basis functions were removed due to overfitting during the backward training. Table 2 shows the models results for BPANN and MARS.

The basis functions equations used in the final MARS model for both training and testing dataset is tabulated in Table 3.

Table 2. Models result for MARS and BPANN (units in metres)

\begin{tabular}{|l|c|c|c|c|}
\hline \multirow{2}{*}{ PCI } & \multicolumn{2}{|c|}{ BPANN } & \multicolumn{2}{c|}{ MARS } \\
\cline { 2 - 5 } & $M S E$ & $R$ & $M S E$ & $R$ \\
\hline Training & $6.1017 \times 10^{-06}$ & 0.7389 & $6.1303 \times 10^{-07}$ & 0.7020 \\
\hline Testing & $1.3249 \times 10^{-04}$ & -0.6067 & $2.2052 \times 10^{-06}$ & 0.6570 \\
\hline
\end{tabular}

The developed MARS and MLR equations for predicting the solid tides effects is shown by Equations (17) and (18) respectively.

$$
\begin{aligned}
d s(i) & =0.014932+0.0206217 \times B F 3-0.0474497 \times B F 9 \\
& -0.0291603 \times B F 13+0.024131 \times B F 15 \\
& -0.0583432 \times B F 17
\end{aligned}
$$




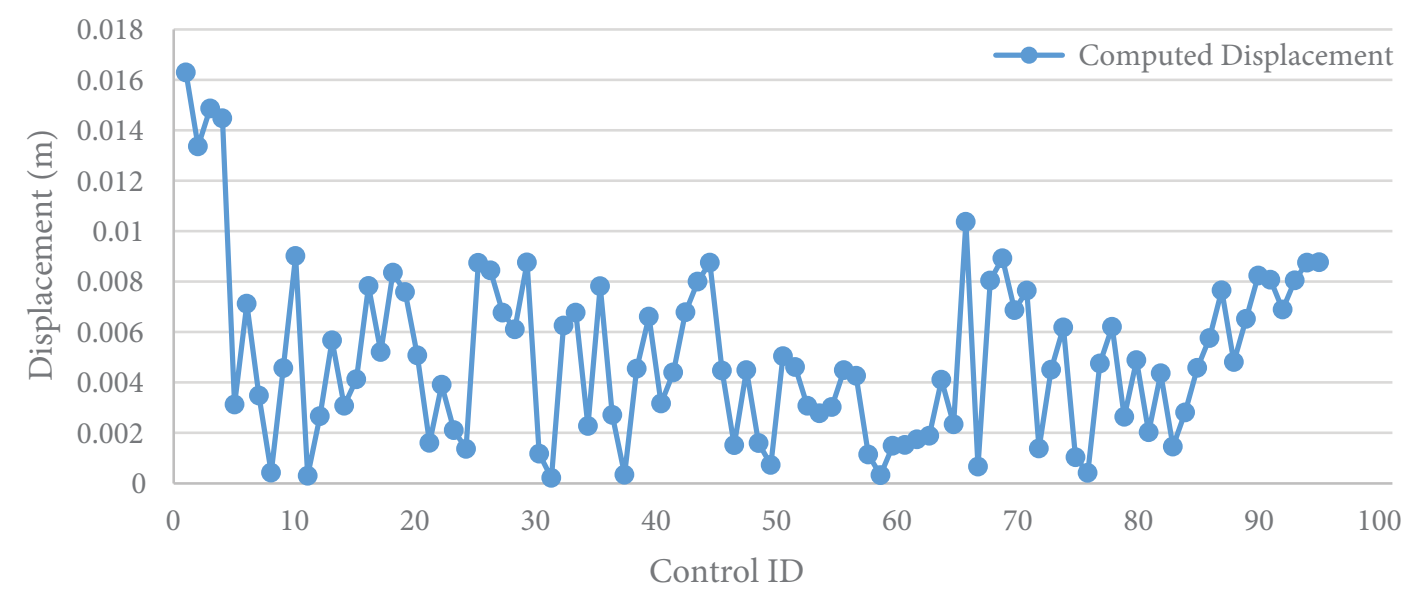

Figure 2. Graph of computed displacement for the six regions

Table 3. Basis Functions Equations for the MARS Model

\begin{tabular}{|c|c|}
\hline Basis Functions & Equations \\
\hline BF3 & $\max \left(0, L_{\left.O N G_{i, j}-2.71766\right)}\right.$ \\
\hline BF9 & $\max \left(0,2.71766-L_{O N G}\right)$ \\
\hline BF13 & $\max \left(0, L A T_{i, j}-6.79333\right)$ \\
\hline BF15 & $\max \left(0, L A T_{i, j}-6.56500\right)$ \\
\hline BF17 & $\max \left(0, L O N G_{i, j}-7.90000\right)$ \\
\hline
\end{tabular}

$$
\begin{aligned}
d s(i)= & -0.0000654+0.00103 \times L O N G_{i, j}+ \\
& 0.000605 \times L A T_{i, j} .
\end{aligned}
$$

Table 4 shows a sample of the results obtained from the geometric computation using Equation (10) and the predicted values by the MARS and BPANN models. From Figure 3, it was observed that both models produce satisfactory results in estimating the tides effects. This implies that there is a good correlation between the independent variables and the dependent variables.

Table 4. Sample of Results Computed and Predicted (Units in metres)

\begin{tabular}{|c|c|c|c|c|c|}
\hline ID & Longitude & Latitude & $\begin{array}{c}\text { Computed } \\
\text { Displa- } \\
\text { cement }\end{array}$ & MARS & BPANN \\
\hline PT 1 & 3.1000 & 6.9417 & 0.0163 & 0.0141 & 0.0157 \\
\hline PT 2 & 2.8600 & 6.7267 & 0.0134 & 0.0097 & 0.0109 \\
\hline PT 3 & 2.9017 & 6.7650 & 0.0149 & 0.0112 & 0.0117 \\
\hline PT 4 & 3.0400 & 6.7933 & 0.0145 & 0.0140 & 0.0129 \\
\hline PT 5 & 2.9033 & 6.5650 & 0.0031 & 0.0064 & 0.0084 \\
\hline PT 6 & 3.1067 & 6.6000 & 0.0071 & 0.0103 & 0.0099 \\
\hline PT 7 & 2.5900 & 6.4150 & 0.0035 & 0.0038 & 0.0050 \\
\hline PT 8 & 2.7467 & 6.4133 & 0.0004 & 0.0041 & 0.0056 \\
\hline PT 9 & 2.9317 & 6.4233 & 0.0046 & 0.0068 & 0.0065 \\
\hline PT 10 & 3.0500 & 6.4233 & 0.0090 & 0.0086 & 0.0070 \\
\hline
\end{tabular}

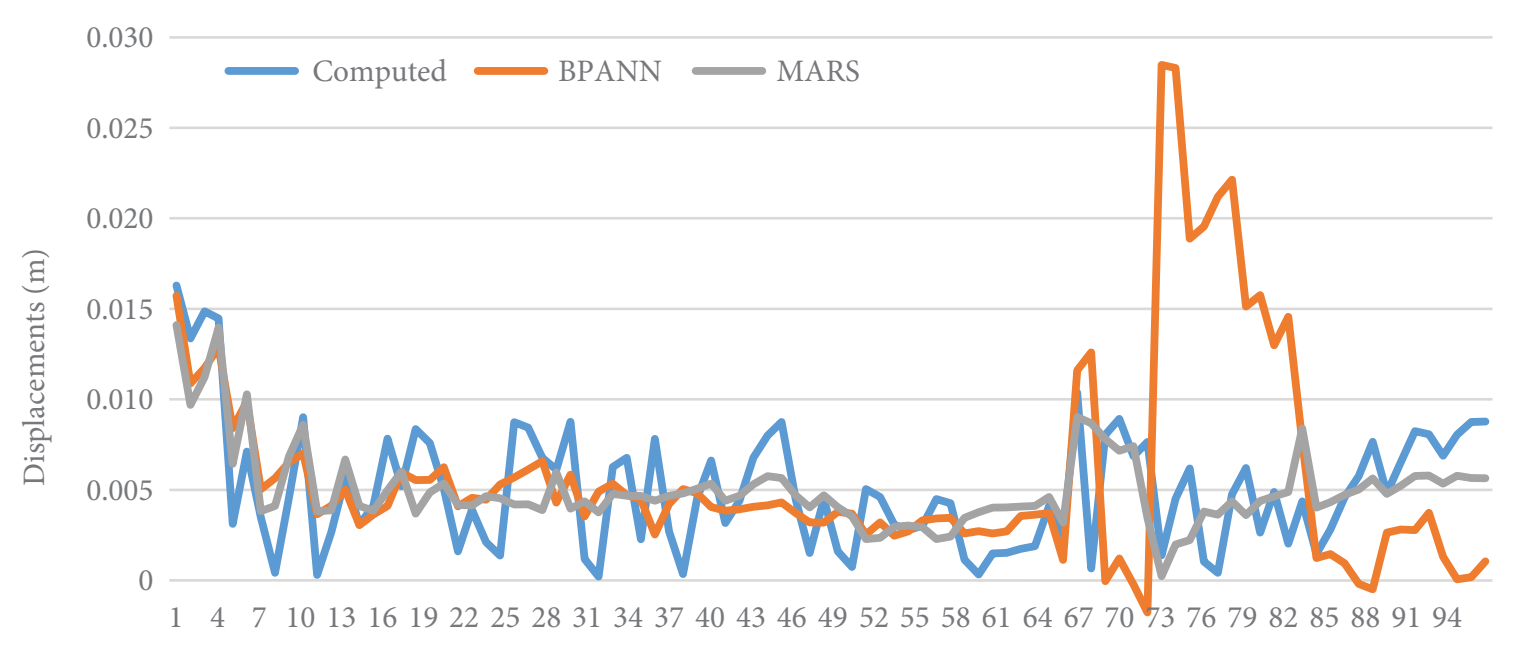

Figure 3. Composite Graph of Computed Displacement and Predicted MARS and BPANN Models 
End of Table 4

\begin{tabular}{|c|c|c|c|c|c|}
\hline ID & Longitude & Latitude & $\begin{array}{c}\text { Computed } \\
\text { Displa- } \\
\text { cement }\end{array}$ & MARS & BPANN \\
\hline PT 11 & 2.6050 & 6.2100 & 0.0003 & 0.0038 & 0.0036 \\
\hline PT 12 & 2.7300 & 6.2367 & 0.0027 & 0.0039 & 0.0041 \\
\hline PT 13 & 2.9200 & 6.2783 & 0.0057 & 0.0067 & 0.0050 \\
\hline PT 14 & 2.3267 & 6.1033 & 0.0031 & 0.0041 & 0.0030 \\
\hline PT 15 & 2.5750 & 6.0833 & 0.0041 & 0.0038 & 0.0036 \\
\hline PT 16 & 2.8050 & 6.1067 & 0.0078 & 0.0050 & 0.0041 \\
\hline PT 17 & 2.8750 & 5.8088 & 0.0052 & 0.0060 & 0.0060 \\
\hline PT 18 & 2.7177 & 5.8340 & 0.0084 & 0.0037 & 0.0055 \\
\hline PT 19 & 2.7967 & 5.8467 & 0.0076 & 0.0049 & 0.0055 \\
\hline PT 20 & 2.8307 & 5.7617 & 0.0051 & 0.0054 & 0.0063 \\
\hline
\end{tabular}

The statistical performance of MARS and BPANN are represented by Figures 4 and 5. The two models are good predictive tools in quantifying tidal effects based on geographic locations within the study area.

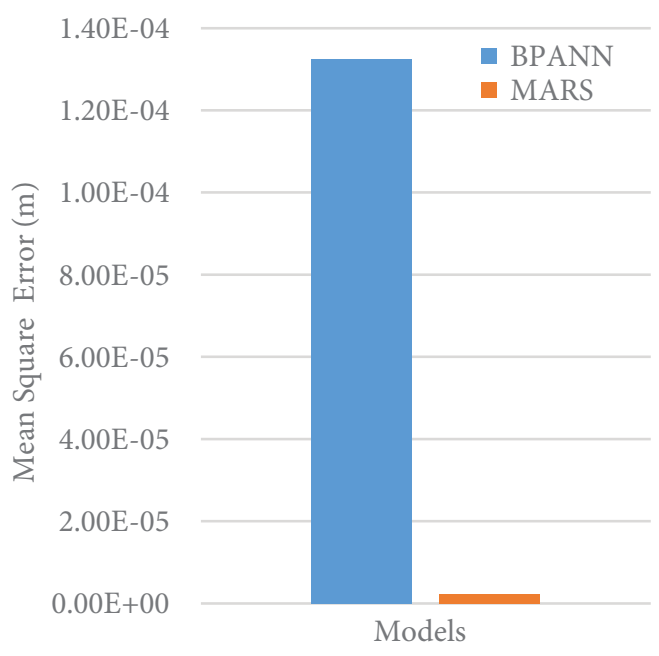

Figure 4. Graph of MSE for MARS AND BPANN

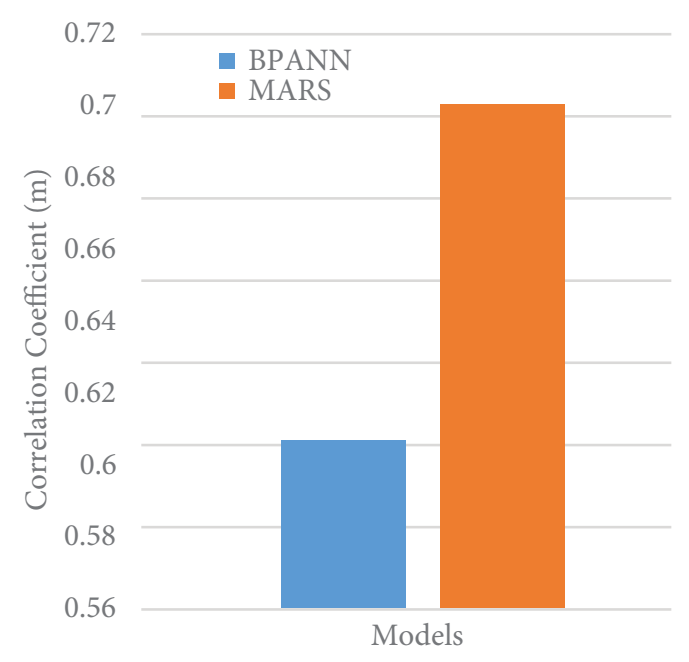

Figure 5. Graph of R for MARS and BPANN

\section{Conclusions}

The solid earth tides cause deformation of both natural and engineering structures. It is important to quantify the tidal effect and incorporate it in periodic deformation monitoring of structures. Tidal effects introduce noise position of structures at a magnitude which shows that without incorporating into proper monitoring of higher engineering structures will result in the likely future casualties which will cause loss of lives and properties. The aim of this present study was to quantify the effects of tides on the earth crust and see its implementation in geodetic deformation modelling through numerical quantities. This study is the first time in Ghana by utilizing MARS, BPANN and MLR models both theoretically and practically in quantifying solid earth tides effects for geodetic deformation modelling. Through the analysis of the results, we conclude that deformation of structures cannot be attributed to mining activities or poor monitoring of highly engineering structures. The external bodies together with gravity (invisible force) have an influence on the stationary positioning of structure and need to be incorporated into our daily monitoring of high engineering structures. Based on the finding in this study MARS, BPANN and MLR models can be used as a realistic alternative technique in quantifying solid earth tides. MARS outperform BPANN and MLR in quantifying the solid earth tides in terms of MSE and R. Geodesist and geotechnical engineers in Ghana can use the developed equations in estimating tidal values for deformation modelling. This study creates the opportunity for geospatial professionals in Ghana to realize the need to quantify tidal values into our daily deformation monitoring. Also, MARS, BPANN and MLR can be used as a robust tool in geodetic deformation modelling.

\section{Acknowledgements}

The authors appreciate some staffs of Survey and Mapping Division of the Lands Commission, Ghana for their support.

\section{Funding}

There were no funding from external source. The authors used their personal funds to finance this research.

\section{Authors' contributions}

YI carried out data collection and the BPANN, MARS models and developed the codes. BKB developed models performance assessment and data analysis and helped to draft the manuscript. Both authors read and approved the final manuscript.

\section{Disclosure statement}

The Principal Author and co-author, declare that there is no any potential conflict of interests in this work. The 
work does not infringe any copyright, proprietary right or any other right of any third party; and the Author and co-author are the sole owners of the work.

\section{References}

Agnew, D. C. (2007). Earth tide (Published Report, pp. 163-191). University of California, San Diego, CA, USA, Elsevier.

Anane, O. E. (2015). Assessing the methods of estimating ellipsoidal height for a local geodetic network (Unpublished BSc Thesis). University of Mines and Technology, Tarkwa, Ghana.

Beltrami, G. M. (2008). An ANN algorithm for automatic, real-time tsunami detection in deep-sea level measurements, Ocean Engineering, 35(5-6), 572-587.

https://doi.org/10.1016/j.oceaneng.2007.11.009

Craven, P., \& Wahba, G. (1979). Smoothing noisy data with spline function: Estimating the correct degree of smoothing by the method of generalized cross-validation. Numerische Mathematik, 31, 317-403. https://doi.org/10.1007/BF01404567

Durmaz, M., \& Karslioglu, M. O. (2011). Non-parametric regional VTEC modelling with Multivariate Adaptive Regression B-Splines. Advances in Space Research, 48, 1523-1530. https://doi.org/10.1016/j.asr.2011.06.031

Friedman, J. H. (1991). Multivariate adaptive regression splines. Annals Statistics, 19, 1-67.

https://doi.org/10.1214/aos/1176347963

Heping, S., Bernard, D., Houze, X., Leslie, V., Jianqiao, X., \& Jiangcun, Z. (2005). Adaptability of the ocean and earth tidal models based on global observations of the superconducting gravimeters. Science in China Series D: Earth Sciences, 48(11), 1859-1869. https://doi.org/10.1360/04yd0071

Kalman, R. E. (1960). A new approach to linear filtering and prediction problems. Transactions of the ASME - Journal of Basic Engineering, 82(1), 35-45.

https://doi.org/10.1115/1.3662552

Kolvankar, V. G., Deshpande, S. S., Manjre, A. S., Pansare, More, S. S., \& Thakur, N. (2010). Lunar periodicities and earthquakes. New Concepts in Global Tectonics Newsletter, (56), 32-49.

Kurinsky, N. (2013). Tidal prediction and compensation in aLIGO (Final Report, pp. 1-58). Tufts University, LIGO Hanford Observatory.

Kutoglu, H. S. (2006). Artificial neural networks versus surface polynomials for determination of local geoid. Paper presented at 1st International Gravity Symposium, Istanbul, Turkey.

Lu, Z., Qu, Y., \& Qiao, S. (2014). Geodesy: Introduction to Geodetic Datum and Geodetic Systems. Springer. https://doi.org/10.1007/978-3-642-41245-5_3

Mendieta, C. (2001). A comparison of artificial neural networks models for predicting tide levels (Master Thesis). Texas A \& M University-Corpus Christi.

Mohammed, A. S. (2015). Performance assessment of the methods used in transformation from cartesian coordinates to geodetic coordinates (Unpublished BSc report). University of Mines and Technology, Tarkwa, Ghana.

Mueller, V. A., \& Hemond, F. H. (2013). Extended artificial neural networks: in-corporation of a priori chemical knowledge enables use of ion selective electrodes for in-situ measure- ment of ions at environmental relevant levels. Talenta, 117, 112-118. https://doi.org/10.1016/j.talanta.2013.08.045

Munk, W. H., \& Cartwright, D. E. (1966). Tidal spectroscopy and predication. Philosophical Transactions of the Royal Society of London, 259(1105), 533-581. https://doi.org/10.1098/rsta.1966.0024

Pavlis, K. N., Holmes, S. A., Kenyon, S. C., \& Factor, J. K. (2008). An earth gravitational model to degree 2160. In EGU General Assembly 2008 (pp. 1-5). Vienna, Austria. https://doi.org/10.1190/1.3063757

Rafiq, M., \& Santos, M. (2004). Study of Eastern Canadian coastal site displacement due to ocean tide loading using a GPS network in Atlantic Canada. In Joint AGU/CCU Scientific Meeting, Montreal, Canada.

Samui, P., \& Kim, D. (2012). Modelling of reservoir-induced earthquakes: a multivariate adaptive regression spline. Journal of Geophysics and Engineering, 9, 494-497. https://doi.org/10.1088/1742-2132/9/5/494

Samui, P., \& Kothari, D. P. (2012). A multivariate adaptive regression spline approach for prediction of maximum shear modulus $\left(\mathrm{G}_{\max }\right)$ and minimum damping ratio (£ $\left.\mathrm{min}\right)$. Engineering Journal, 16(5), 1-10. https://doi.org/10.4186/ej.2012.16.5.69

Samui, P. (2013). Multivariate Adaptive Regression Spline (MARS) for prediction of elastic modulus of jointed rock mass. Geotechnical and Geological Engineering, 31, 249-253. https://doi.org/10.1007/s10706-012-9584-4

Siek, M., \& Solomatine, D. P. (2010). Nonlinear chaotic model for predicting storm surges. Nonlinear Processes in Geophysics, 17, 405-420. https://doi.org/10.5194/npg-17-405-2010

Straser, V. (2010). Variations in gravitational field, tidal force, electromagnetic waves and earthquakes. New Concepts in Global Tectonics Newsletter, (57), 98-108.

Torge, W. (1991). Geodesy (2 ed.). Walter de Gruyter. https://doi.org/10.1515/9783111542683

Vaziri, M. (1997). Predicting Caspian Sea surface water level by ANN and ARIMA models. Journal of Waterway, Port, Coastal and Ocean Engineering, 123, 158-162. https://doi.org/10.1061/(ASCE)0733-950X(1997)123:4(158)

Yakubu, I. (2008). Local area deformation monitoring a multi GPS receiver network system approach-a case study (MPhil Thesis). University of Mines and Technology, Tarkwa, Ghana.

Yegnanarayana, B. (2005). Artificial neural networks. PrenticeHall of India Private Limited.

Zabihi, M., Pourghasemi, H. R., Pourtjhi, Z. S., \& Behzadfar, M. (2016). GIS-based multivariate adaptive regression spline and random forest models for groundwater potential mapping in Iran. Environmental Earth Sciences, 75(665), 646-665. https://doi.org/10.1007/s12665-016-5424-9

Ziggah, Y. Y., Youjian, H., Tierra, A., Konate, A. A., \& Hui, Z. (2016a). Performance evaluation of artificial neural networks for planimetric coordinate transformation - A case study, Ghana. Arabian Journal of Geosciences, 9, 698-714. https://doi.org/10.1007/s12517-016-2729-7

Ziggah, Y. Y., Youjian, H., Yu, X., \& Basommi, L. P. (2016b). Capability of artificial neural network for forward conversion of geodetic coordinates $(\Phi, \lambda, \mathrm{h})$ to cartesian coordinates $(\mathrm{X}, \mathrm{Y}$, Z). Mathematical Geosciences, 48, 687-721. https://doi.org/10.1007/s11004-016-9638-x 